\title{
Nuclear BCL-10 expression is common in lymphoplasmacytic lymphoma/Waldenström macroglobulinemia and does not correlate with p65 NF- $\kappa$ B activation
}

\author{
Mihai Merzianu ${ }^{1}$, Liuyan Jiang ${ }^{2}$, Pei Lin ${ }^{1}$, Xuemei Wang ${ }^{3}$, Donna M Weber ${ }^{4}$, \\ Saroj Vadhan-Raj ${ }^{5}$, Martin H Nguyen ${ }^{1}$, L Jeffrey Medeiros ${ }^{1}$ and Carlos E Bueso-Ramos ${ }^{1}$ \\ ${ }^{1}$ Department of Hematopathology, The University of Texas MD Anderson Cancer Center, Houston, TX, USA; \\ ${ }^{2}$ Department of Pathology, The University of Texas MD Anderson Cancer Center, Houston, TX, USA; \\ ${ }^{3}$ Department of Biostatistics and Applied Mathematics, The University of Texas MD Anderson Cancer Center, \\ Houston, TX, USA; ${ }^{4}$ Department of Lymphoma, The University of Texas MD Anderson Cancer Center, \\ Houston, TX, USA and ${ }^{5}$ Department of Cytokine and Supportive Oncology, The University of Texas MD \\ Anderson Cancer Center, Houston, TX, USA
}

\begin{abstract}
B-cell lymphoma 10 (BCL-10) is expressed in the cytoplasm of normal germinal center and marginal zone Bcells and is involved in lymphocyte development and activation. Aberrant nuclear expression of BCL-10 occurs in a subset of extranodal marginal zone B-cell lymphomas (MALT lymphomas), primarily those with the $t(1 ; 14)(p 22 ; q 32)$ or $t(11 ; 18)(q 21 ; q 21)$. Little is known about $B C L-10$ expression in lymphoplasmacytic lymphoma/ Waldenström macroglobulinemia (LPL/WM). We assessed for BCL-10 in 51 bone marrow (BM) specimens involved by LPL/WM using immunohistochemical methods. All patients had monoclonal IgM in serum. Extent of BM involvement was assessed using PAX-5/BSAP and CD20 immunostains and the pattern and percentage of B-cells positive for BCL-10 was determined. The p65 subunit of nuclear factor-kappa B (NF- $k$ B), a molecule downstream of BCL-10, was also assessed immunohistochemically. Nuclear BCL-10 staining was present in 28/ $51(55 \%)$ specimens. BCL-10 expression correlated with greater extent of BM involvement $(P=0.001)$, but did not correlate with serum IgM paraprotein levels, type of immunoglobulin light chain, or clinical variables. Nuclear expression of the p65 subunit of NF- $\kappa$ B was detected in $17 / 50(34 \%)$ specimens, suggesting that NF- $\kappa$ B is active in a subset of LPL/WM. p65 NF- $\kappa$ B activation did not correlate with nuclear BCL-10 immunostaining. Cytogenetic analysis in 29 cases showed no evidence of the $t(1 ; 14)$ or $t(11 ; 18)$. These results indicate that nuclear BCL-10 expression is common in LPL/WM and does not correlate with MALT lymphoma-associated translocations or p65 NF- $\kappa$ B nuclear staining.
\end{abstract}

Modern Pathology (2006) 19, 891-898. doi:10.1038/modpathol.3800609; published online 21 April 2006

Keywords: BCL10; p65; NF- $\kappa$ B; lymphoplasmacytic lymphoma; Waldenström macroglobulinemia

Waldenström macroglobulinemia (WM) has been defined as a neoplasm of $B$ lymphocytes that involves the bone marrow (BM) and is associated with monoclonal immunoglobulin $\mathrm{M}$ (IgM) in serum. ${ }^{1}$ Most patients with the clinical and laboratory findings of WM have been classified as having lymphoplasmacytic lymphoma (LPL), and thus LPL

Correspondence: Dr CE Bueso-Ramos, MD, PhD, Department of Hematopathology, Unit 72, The University of Texas MD Anderson Cancer Center, 1515 Holcombe Blvd., Houston, TX 77030, USA. E-mail: cbueso@mdanderson.org

Received 23 January 2006; revised 2 March 2006; accepted 10 March 2006; published online 21 April 2006 and WM are linked in the World Health Organization classification. ${ }^{2}$

Lymphoplasmacytic lymphoma/Waldenström macroglobulinemia constitutes approximately $2 \%$ of all hematopoietic neoplasms. ${ }^{1,3-5}$ Patients are usually older adults (median age, 63 years), and there is a slight male preponderance. ${ }^{5,6}$ The serum concentration of IgM paraprotein varies widely in WM; thus presence of monoclonal IgM in serum, irrespective of level, and BM involvement are considered as two of the diagnostic requirements in the definition of WM as suggested by the Second International Workshop on Waldenström Macroglobulinemia (Table 1). ${ }^{1}$ The clinical features are highly variable, and many patients are asymptomatic. ${ }^{5}$ Clinically, a 
Table 1 Diagnostic criteria for Waldenström macroglobulinemia recommended by the Second International Workshop on Waldenström macroglobulinemia

Criteria

- IgM monoclonal gammopathy of any concentration

- Bone marrow infiltration by small lymphocytes showing plasmacytoid/plasma cell differentiation

- Intertrabecular pattern of bone marrow infiltration

- Surface IgM+, CD5 \pm , CD10-, CD19+, CD20+, CD22+, CD23-, CD25+, CD27+, FMC7+, CD103-, CD138- immunophenotype.

subset of patients has evidence of lymphadenopathy, hepatosplenomegaly, anemia, cytopenias, ${ }^{3,7-10}$ and hyperviscosity syndrome. ${ }^{5}$ The latter, however, is uncommon in patients with LPL/WM in our experience. ${ }^{11}$ Patients with LPL/WM usually have clinically indolent disease, with a median survival of 5 years, and up to $40 \%$ of patients survive more than 10 years or die from unrelated causes. ${ }^{6,12}$ Some patients, however, can develop transformation to diffuse large B-cell lymphoma. ${ }^{13}$ LPL/WM is generally incurable with available therapy.

$B C L-10$ is a proapoptic gene in vitro with a role in lymphocyte maturation, differentiation, and activation. ${ }^{14}$ BCL-10 cytoplasmic protein in humans is relatively restricted to lymphocytes, being expressed in B-cells with the strongest intensity in the germinal center, and less intensely in the marginal zone. ${ }^{14}$ Strong BCL-10 nuclear expression was first described in extranodal marginal zone B-cell lymphomas (MALT lymphoma) with the $\mathrm{t}(1 ; 14)(\mathrm{p} 22 ; \mathrm{q} 32)$ and the $B C L-10$ gene was subsequently cloned and breakpoints identified. $.^{15,16} \mathrm{Mod}-$ erate nuclear expression of BCL-10 also has been reported in MALT lymphomas with the $t(11 ; 18)$ and strong cytoplasmic expression occurs in MALT lymphomas with the $\mathrm{t}(14 ; 18)$ involving the MALT1 gene. ${ }^{17-20}$

$\mathrm{NF}-\kappa \mathrm{B}$ is a transcription factor composed of five proteins (p50, p52, p65, c-Rel, and RelB) with a role in apoptosis regulation, cell proliferation, survival, and metastasis. ${ }^{21,22} \mathrm{NF}-\kappa \mathrm{B}$, when inactive, is located in the cytoplasm and is bound to an inhibitory molecule, I kappa B ( $\mathrm{I} \kappa \mathrm{B})$. Upon phosphorylation, ubiquitination, and degradation of $\mathrm{I} \kappa \mathrm{B}, \mathrm{NF}-\kappa \mathrm{B}$ is released into the cytosol, translocates into the nucleus, and transactivates several genes. Constitutive activation of NF- $\kappa \mathrm{B}$ is implicated in oncogenesis of many neoplasms. ${ }^{23-26}$ The presence of NF- $\kappa$ B proteins in the nucleus of the neoplastic cells is a harbinger of activation of the NF- $\kappa \mathrm{B}$ complex.

In this study, we evaluated for nuclear expression of BCL-10 by immunohistochemistry in cases of LPL/WM. Furthermore, nuclear expression of p65 was used as a surrogate of NF- $\kappa \mathrm{B}$ complex activation. Correlation between BCL-10, p65, available cytogenetic data, and various clinical and pathologic parameters was also performed.

\section{Materials and methods}

\section{Tissue Specimens}

From our archives, we retrieved BM specimens involved by LPL/WM. Approval for this study from the institutional review board was obtained. LPL/ WM was defined on the basis of a combination of laboratory, clinical, morphologic, and immunophenotypic criteria as described by the World Health Organization $^{2}$ and the International Workshop on WM guidelines (Table 1). ${ }^{1}$ The inclusion criteria for cases of LPL/WM in this study included IgM paraprotein in serum, presence of lymphoma in the BM composed of small lymphocytes of which a subset had plasmacytoid differentiation, and results of immunophenotypic studies (flow cytometry and/or immunohistochemistry) that demonstrated a monoclonal B-cell population positive for pan-Bcell antigens (CD19 or CD20) and negative for CD5 and CD10. We also excluded neoplasms that were associated with IgA or IgG serum paraprotein, as well as patients with IgM lymphoproliferative disorders associated with nodal or bulky splenic disease.

We also assessed a control group of formalinfixed, paraffin-embedded BM specimens including 10 normal BM and 64 lymphomas of various types: 14 MALT lymphoma (four with $t(11 ; 18)$ ), two nodal marginal zone B-cell lymphoma, one splenic marginal zone B-cell lymphoma, 11 follicular lymphoma, 20 chronic lymphocytic leukemia/small lymphocytic lymphoma, 11 mantle cell lymphoma, and five diffuse large B-cell lymphoma.

\section{Immunohistochemistry}

We performed immunostaining using 5 - $\mu \mathrm{m}$-thick, formalin-fixed, paraffin-embedded tissue sections, heat-induced epitope retrieval, and the Envision + system (Dakocytomation, Carpinteria, CA, USA) according to the manufacturer's recommendations. The staining was carried out using Autostainer Plus (Dakocytomation). The washing buffer used was $0.05-\mathrm{M}$ Tris-buffered saline supplemented with $0.05 \%$ Tween.

\section{Single antibody staining}

The following monoclonal antibodies were used: CD20 (Dako; 1:300), BCL-10 (Zymed, San Francisco, CA, USA; 1:40), NF- $\kappa$ B antibody p65, rabbit (polyclonal GTX27970, GeneTex Inc., San Antonio, TX, USA; 1:100), and PAX-5/BSAP (BD Transduction Laboratories, San Diego, CA, USA; 1:20).

Sections were first deparaffinized in xylene and rehydrated through graded concentrations of alcohol. Sections were heated in a steamer (Black \& Decker HS2776 Type 1, Miami Lakes, FL, USA) for $20 \mathrm{~min}$ in target retrieval solution (Dakocytomation) for antigen retrieval. The activity of endogenous peroxidase was blocked with peroxidase-blocking 
reagent (Dakocytomation) for $5 \mathrm{~min}$. After washing the sections with buffer, we incubated them with primary antibodies for $30 \mathrm{~min}$ at $23^{\circ} \mathrm{C}$. Sections were then washed again with buffer.

The Envision + HRP system (Dakocytomation) was used to visualize antigen expression. Diaminobenzidine tetrahydrochloride was used as the chromogen, and all sections were counterstained with hematoxylin. A sample of MALT lymphoma with the $t(11 ; 18)$ was used as a positive control for BCL-10 expression. A case of breast carcinoma was used as a positive control for p65 NF- $\kappa \mathrm{B}$ staining.

\section{Double staining}

The first antibody, BCL-10, was applied as described above. After visualizing BCL-10 expression with diaminobenzidine tetrahydrochloride, we rewashed the section in buffer. Double-stain block (Dakocytomation) was applied for $3 \mathrm{~min}$, the sections were incubated for $30 \mathrm{~min}$ at $23^{\circ} \mathrm{C}$ with the second antibody, CD20, and then rewashed. The expression of CD20 was visualized with Fast-Red substrate (Dakocytomation). All sections were then counterstained with hematoxylin.

\section{Antibody Expression}

Three observers (MM, LJM, and CBR) without knowledge of the clinical data evaluated the immunohistochemical results. Double staining was used only for the qualitative evaluation of the coexpression pattern. Cell counts were performed only in the single antibody-stained tissue sections. We used $\times 400$ magnification to determine the percentage of tumor cells that exhibited positive immunoreactivity. At least 1000 neoplastic cells were counted in each case. The presence of membrane staining for CD20 and nuclear staining for PAX-5/BSAP, p65 NF- $\kappa \mathrm{B}$, and BCL-10 were scored as positive. For BCL-10 positive nuclear staining, we used a cutoff of $\geq 20 \%$ to classify tumors as having low and high expression.

For p65 NF- $\kappa \mathrm{B}$, the intensity of nuclear immunohistochemical staining was evaluated as follows: - negative, + focal expression in $<5 \%$ of cells, and ++ diffuse expression in $\geq 5 \%$ of cells. Samples with ++ staining were classified as the 'highexpression group', and those with - or + were classified as the 'low-expression group'. We used only two categories for statistical analysis: positive $(\geq 5 \%$ nuclei positive) and negative $(<5 \%$ nuclei positive).

PAX-5/BSAP expression was used in conjunction with hematoxylin and eosin-stained sections to evaluate the extent and pattern of BM involvement.

\section{Clinical and Cytogenetic Data}

Clinical follow-up was obtained by reviewing the medical records of the patients. Most patients had been followed-up with routine monitoring of serum IgM levels, BM aspiration and biopsy, or tissue biopsy when clinically indicated. The patients' demographic data, time from original diagnosis, concentration of serum monoclonal IgM at time of biopsy (determined by nephelometry), and presence or absence of therapy were retrieved, statistically analyzed, and tabulated. Results of conventional G-band karyotype analysis were available for 29 of the 51 samples.

\section{Statistical Analysis}

Fisher's exact test and Wilcoxon rank-sum test were used to determine the association among BCL-10, p65 and various clinicopathologic parameters. $P$-values $<0.05$ were deemed as statistically significant. All analyses were carried out in S-plus software (Insightful Corp., Seattle, WA).

\section{Results}

We assessed $51 \mathrm{BM}$ specimens involved by LPL/WM obtained from 47 patients. There were 28 men and 19 women with a mean age of 63 years (range, 39-80 years). The average percentage of $\mathrm{BM}$ involvement per biopsy specimen was 48\% (range 5-90\%). Monoclonal IgM was present in the serum of all patients (range $0.2-10 \mathrm{~g} / \mathrm{dl}$; mean $3.7 \mathrm{~g} / \mathrm{dl}$ ). In all, 36 of $47(77 \%)$ patients had $\kappa$ immunoglobulin lightchain restriction, and $10(21 \%)$ patients had $\lambda$ lightchain restriction. One patient had a biclonal phenotype, with both $\kappa$ and $\lambda$ light chains.

From the time of initial biopsy, the average duration of disease was 2.5 years (range, 1 month10 years). In all, 24 patients had received some form of therapy, and 23 had not. We compared these two groups and found no significant difference between the clinical and pathologic variables. The only exception was an expected correlation between time with disease and therapy $(P=0.0002$, Table 2). As these groups had no important differences, we combined them for the data analysis (Table 2). The results of BCL-10 expression in B-cell lymphomas and normal bone marrow were summarized in Table 3.

BCL-10 cytoplasmic expression was present in virtually all cases of LPL/WM and therefore was not quantitatively analyzed in this study. It did parallel the expression of CD20 and PAX-5/BSAP, allowing the observer to evaluate the extent and pattern of BM involvement (Figure 1). Nuclear staining for BCL-10 was present in 28 of 51 (55\%) samples. We used the double-staining technique for BCL-10 and CD20 to confirm the presence of BCL-10 expression in the neoplastic cells. Nuclear staining intensity in most cases was weak or moderate (Figures 1 and 2). No cases with strong nuclear BCL-10 expression were identified. 
Table 2 Differences between the treated and 'not treated' patient groups

\begin{tabular}{lccc}
\hline Group & Not treated & Treated & P-value \\
\hline $\begin{array}{l}\text { Sex } \\
\text { Female }\end{array}$ & $9(39 \%)$ & $10(42 \%)$ & 1.00 \\
$\quad$ Male & 14 & 14 & \\
$\begin{array}{l}\text { BCL10 } \\
\quad \text { Negative }\end{array}$ & $10(43 \%)$ & $10(42 \%)$ & 1.00 \\
$\quad$ Positive & 13 & 14 & \\
$\begin{array}{l}\text { P65 } \\
\quad \text { Negative }\end{array}$ & $13(59 \%)$ & $16(67 \%)$ & \\
$\quad$ Positive & 9 & 8 & \\
$\begin{array}{l}\text { Light chain } \\
\text { kappa }\end{array}$ & $16(73 \%)$ & $20(83 \%)$ & 0.76 \\
$\quad$ lambda & 6 & 4 & \\
$\begin{array}{l}\text { BM involvement } \\
\text { (\%) }\end{array}$ & $40(5-90)$ & $50(10-90)$ & 0.94 \\
$\begin{array}{l}\text { IgM at biopsy } \\
\text { (mg/dl) }\end{array}$ & $3390(241-10200)$ & $3120(600-8360)$ & 0.99 \\
$\begin{array}{l}\text { Time with } \\
\text { disease (years) }\end{array}$ & $0.5(0.1-7.0)$ & $3.0(0.4-10.0)$ & 0.0002 \\
\hline
\end{tabular}

The figures in each cell represent if not otherwise specified $N(\%)$ or median (range).

Table 3 Summary of BCL-10 expression in B-cell lymphomas and normal bone marrow

\begin{tabular}{lcc}
\hline Pathologic diagnosis & $\begin{array}{c}\text { Number } \\
\text { of cases } \\
\text { (N) }\end{array}$ & $\begin{array}{c}\text { Number (\%) of } \\
\text { cases with } \\
\text { nuclear staining } \\
\text { (cutoff }>20 \%)\end{array}$ \\
\hline $\begin{array}{l}\text { Lymphoplasmacytic lymphoma/ } \\
\text { WM }\end{array}$ & 51 & $28(55 \%)$ \\
MALT lymphoma without t(11;18) & 10 & $3(30 \%)$ \\
MALT lymphoma with t(11;18) & 4 & $4(100 \%)$ \\
Nodal marginal zone B-cell & 2 & $1(50 \%)$ \\
$\begin{array}{l}\text { lymphoma } \\
\text { Splenic marginal zone B-cell }\end{array}$ & 1 & $0(0 \%)$ \\
$\begin{array}{l}\text { lymphoma } \\
\text { Follicular lymphoma }\end{array}$ & 11 & $0(0 \%)$ \\
$\begin{array}{l}\text { Chronic lymphocytic leukemia/ } \\
\text { small lymphocytic lymphoma }\end{array}$ & 20 & $0(0 \%)$ \\
$\begin{array}{l}\text { Mantle cell lymphoma } \\
\text { Diffuse large B-cell lymphoma }\end{array}$ & 11 & $1(9 \%)$ \\
Normal bone marrow & 5 & $0(0 \%)$ \\
\hline & 10 & $0(0 \%)$ \\
\hline
\end{tabular}

Cytoplasmic p65 was expressed in most cases. p65 nuclear expression was identified in 17 of 50 (34\%) specimens (Figure 3a); the double-staining technique for p65 and CD20 was used to confirm p65 expression in the neoplastic cells. Unlike BCL-10 nuclear expression that was accompanied by uniform cytoplasmic staining, p65 cytoplasmic staining was diminished, and in some neoplasms difficult to appreciate, in cases with strong nuclear p65 expression (Figure 3b).

There was no significant association between nuclear expression of BCL-10 and p65 $(P=0.54$,

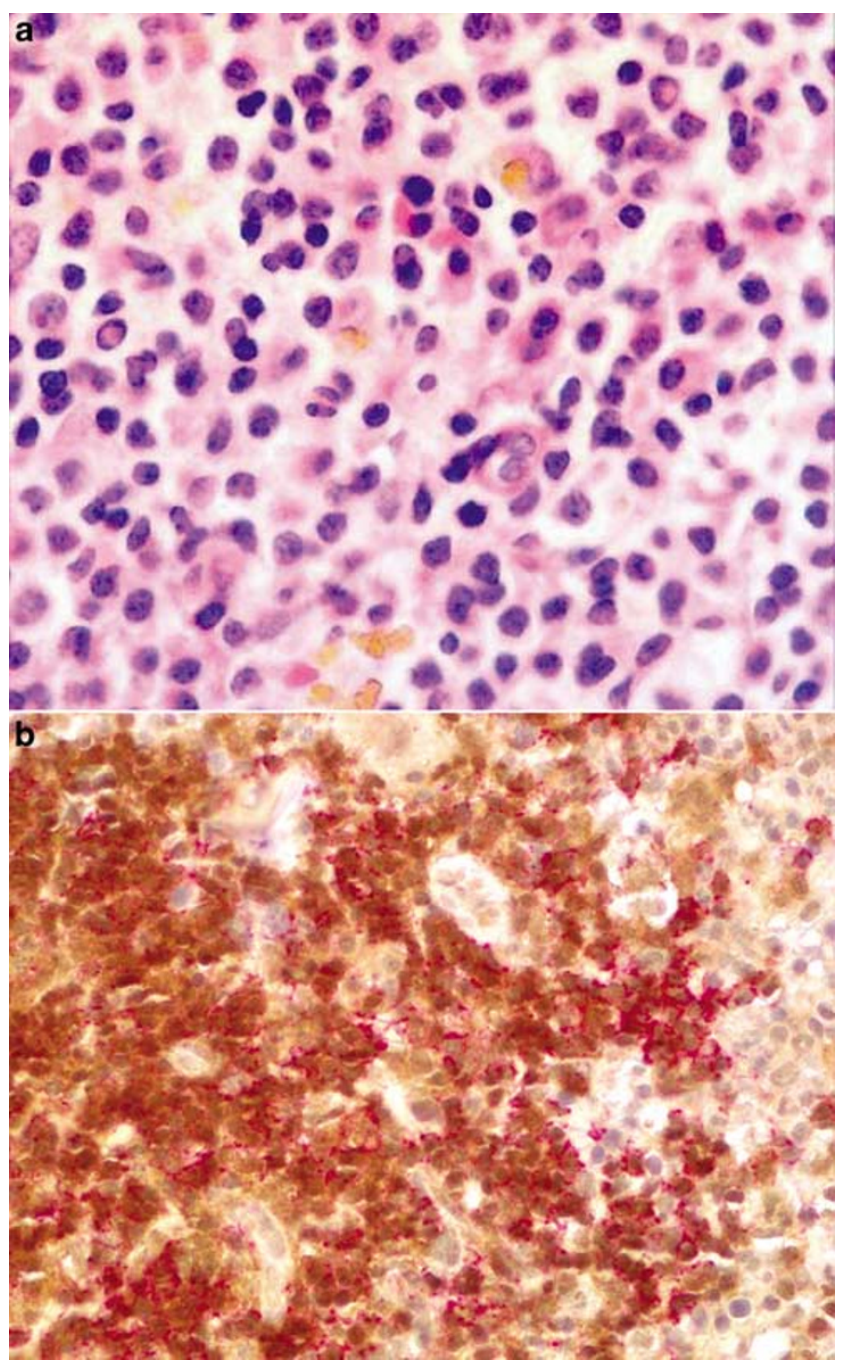

Figure 1 Lymphoplasmacytic lymphoma/Waldenström macroglobulinemia (LPL/WM) diffusely involving BM. (a) Representative field of the neoplasm showing small lymphoma cells. Note the Dutcher bodies in the field (hematoxylin-eosin). (b) Double staining for BCL-10 and CD20 demonstrates nuclear and cytoplasmic BCL-10 expression restricted to the neoplastic cells that are highlighted by the CD20 membranous stain. (a, b. Original magnification for all images is $\times 200$ ).

Fisher's exact test). Nuclear BCL-10 expression statistically correlated with extent of BM involvement. The median percentage of $\mathrm{BM}$ involvement for the BCL-10 negative and positive groups was 25 and 70 , respectively $(P=0.001$, Wilcoxon rank-sum test). Nuclear BCL-10 expression did not correlate with sex, type of immunoglobulin light chain restriction, serum IgM level, and time with disease. Similarly, the associations between nuclear p65 expression and several clinicopathologic variables were assessed. There was a marginal association between nuclear p65 expression and extent of BM involvement. The median percentage of BM involvement for the p65 negative and p65 positive groups was 50 and 30 , respectively $(P=0.09$, Wilcoxon rank-sum test). No correlation between nuclear p65 

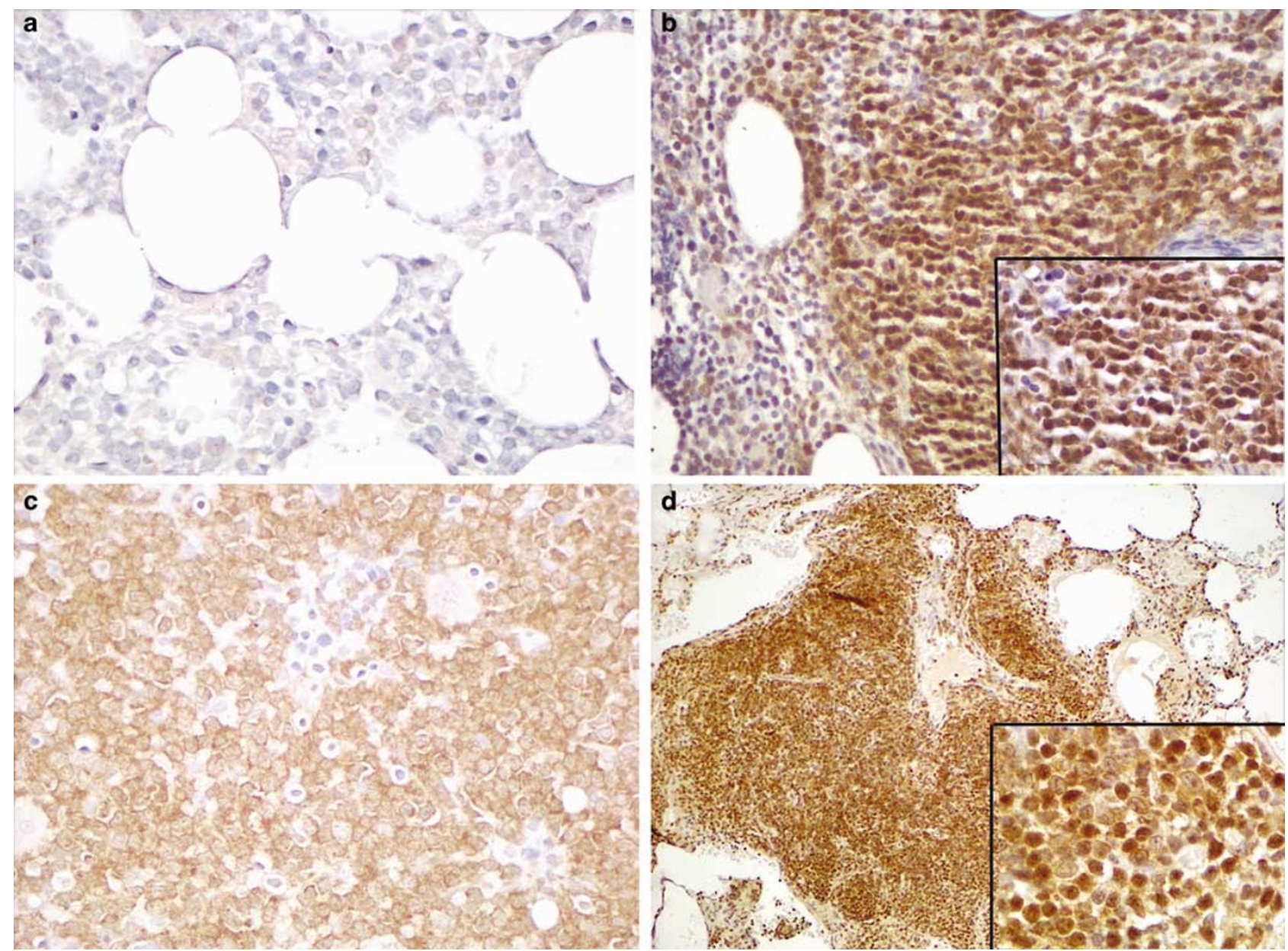

Figure 2 (a) Normal BM biopsy specimen negative for BCL-10 in the hematopoietic elements; a few small mature lymphocytes show weak cytoplasmic staining. (b) In contrast, LPL/WM involving the BM shows extensive nuclear and cytoplasmic BCL-10 expression. (c) Mantle cell lymphoma involving BM with uniform cytoplasmic staining for BCL-10. (d) MALT lymphoma with t(11;18) involving lung shows moderate to strong nuclear staining for BCL-10 (original magnification for all images $\times 200$, except the insets $\times 400$ ).

protein expression and the other variables was identified.

Four patients in this study had a follow-up BM biopsy specimen after a variable interval (9 months10 years). In two patients, BCL-10 nuclear expression was found in the original biopsy specimen but was absent in the follow-up specimen. In one patient, BCL-10 was absent in both the initial and follow-up specimens. In the fourth patient, nuclear expression of BCL-10 was present only in the follow-up biopsy specimen. BCL-10 nuclear staining in these four patients showed some correlation with extent of tumor involvement and no correlation with therapy unless tumor volume decreased. In the two patients in whom the original biopsy specimen was positive for BCL10, in the follow-up biopsy specimen BCL-10 was negative and tumor burden was decreased. No correlation between BCL-10 nuclear staining and extent of BM involvement was observed in the other two patients. All biopsy specimens in this subgroup of patients were negative for nuclear p65 expression.
Conventional cytogenetic studies were performed on BM aspirate specimens of 29 LPL/WM patients. A total of 21 patients had a normal karyotype. Random clonal chromosomal abnormalities were identified in eight patients. None had the $t(1 ; 14)$, $t(11 ; 18), t(14 ; 18)$, or $t(9 ; 14)$, and no other recurrent cytogenetic anomalies were identfied.

\section{Discussion}

The $B C L-10$ gene was first identified by its involvement in the $t(1 ; 14)(p 22 ; q 32)$ in two cases of MALT lymphoma. ${ }^{15,16}$ Via the $\mathrm{t}(1 ; 14)$, the $B C L-10$ gene located on $1 \mathrm{p} 22$ is juxtaposed with and comes under the influence of IgH enhancers on chromosome $14 \mathrm{q} 32$, resulting to BCL-10 overexpression.

In normal B- and T-cells, BCL-10 plays a role in antigen-receptor-mediated activation of the NF- $\kappa \mathrm{B}$ complex ${ }^{14}$ via its binding with MALT1 and other important molecules in this pathway. Physiologically, BCL-10 has an exclusively cytoplasmic 
896

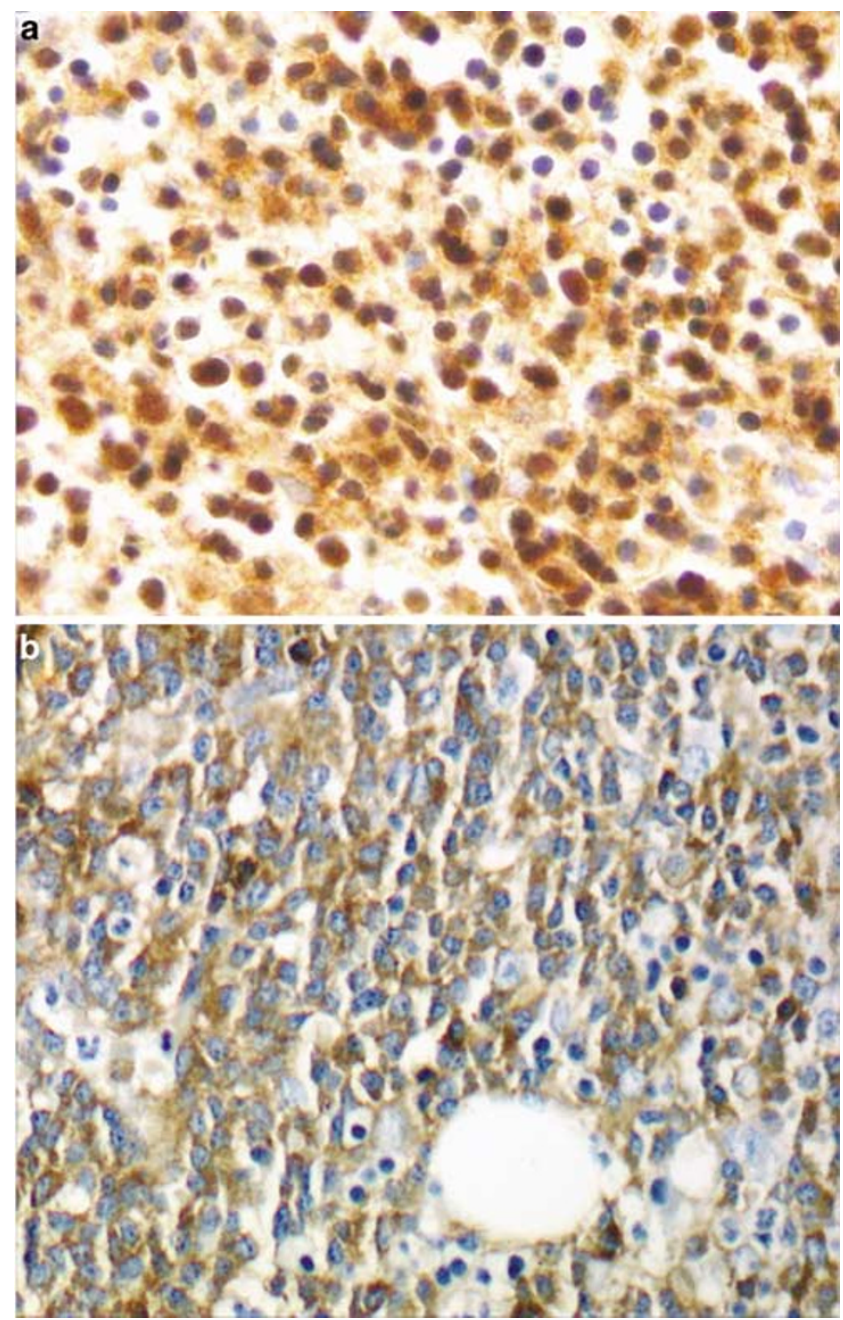

Figure 3 (a, b) Two cases of LPL/WM involving BM. (a) strong nuclear expression of p65 NF- $\kappa \mathrm{B}$, and (b) diminished cytoplasmic staining for p65 NF- $\kappa \mathrm{B}$, (original magnification for all images $\times 200)$.

localization. ${ }^{18}$ In MALT lymphomas, however, BCL10 is aberrantly located in the nucleus of neoplastic cells that carry the $t(1 ; 14)$ and $t(11 ; 18)$, with strong or moderate expression, respectively. In fact, some investigators have used the pattern of BCL-10 immunostaining as a surrogate marker for the presence of these chromosomal translocations in MALT-lymphomas. ${ }^{19,27}$ Currently, the mechanism underlying this aberrant expression pattern is not understood, but $B C L-10$ gene mutation seems an unlikely cause. ${ }^{27-30}$ Moderate nuclear BCL-10 expression, however, has been observed in some cases of MALT lymphoma without the $t(11 ; 18) .^{20,31,32} \mathrm{In}$ addition, other B-cell lymphoproliferative disorders have been reported to express BCL-10 in a nuclear pattern although the staining is of moderate or weak intensity. ${ }^{17,27}$ These findings are controversial, however, as other studies using immunohistochemistry or fluorescence in situ hybridization have failed to confirm BCL-10 nuclear expression in non-MALT lymphomas. ${ }^{27-29}$
In this study, we were interested in assessing BCL-10 expression in LPL/WM, a neoplasm that has been rarely studied for BCL-10 expression in the past, most likely because these neoplasms are rare and primarily involve the BM. We identified aberrant nuclear expression of BCL-10 in more than half of the specimens. Nuclear BCL-10 expression correlated with extent of BM involvement by LPL/WM, but did not correlate with other analyzed variables (age, sex, serum monoclonal IgM level, type of immunoglobulin light chain, and time with disease). As extent of involvement of the BM biopsy specimen is inversely associated with the survival rate of patients with LPL/WM, ${ }^{9}$ nuclear expression of BCL-10 in LPL/WM may have prognostic value although the explanation for this correlation is unknown.

As the biologic significance of nuclear BCL-10 in LPL/WM is unknown, we also assessed for expression of the p65 subunit of NF- $\kappa \mathrm{B}$ to determine if the $\mathrm{NF}-\kappa \mathrm{B}$ pathway is activated. Others have shown that the NF- $\kappa$ B pathway is active in MALT lymphomas, and have suggested that NF- $\kappa \mathrm{B}$ is a common downstream pathway for MALT lymphomas with the $\mathrm{t}(11 ; 18), \mathrm{t}(1 ; 14)$, and $\mathrm{t}(14 ; 18) / I g H-M A L T 1 . \mathrm{NF}-\kappa \mathrm{B}$ is a transcription factor that is composed of five proteins that form heterodimers. The p65 subunit is involved in most of these heterodimers. By detecting p65 expression and location, the results are a reasonable approximation of the activation status of NF- $\kappa \mathrm{B}$. We detected nuclear expression of p65 in approximately one third of cases of LPL/WM. These results indicate that NF- $\kappa \mathrm{B}$ is activated in a subset of LPL/ WM cases. Nuclear p65 expression did not statistically correlate with BCL-10 nuclear staining or other clinicopathologic parameters although there may be a trend suggesting that nuclear p65 expression inversely correlates with extent of BM involvement $(P=0.09)$. As we were unable to find a statistically significant association between p65 and BCL-10, it is unlikely that BCL-10 has a major role in the downstream activation of p65 NF- $\kappa \mathrm{B}$ in LPL/WM. Different molecular mechanisms of NF- $\kappa$ B activation have been proposed. ${ }^{33,34}$ In the canonical or classical pathway, dimers contain either RelA or c-Rel, together with p50. Upon activation by pro-inflammatory cytokines, the IkB proteins are phosphorylated and undergo ubiquitin-dependent degradation by the proteasome, and NF- $\kappa \mathrm{B}$ is translocated to the nucleus where it acts as transcription factor. Members of the tumor necrosis factor family activate the alternative pathway. The dimers contain RelB and p100 subunits. This pathway mainly activates IKK $\alpha$, which phosphorylates p100 that is subsequently undergoes proteolyis to generate the p52 mature form. ${ }^{35,36}$ It is possible that other NF- $\kappa$ B subunits such as C-Rel or RelB are involved in NF- $\kappa$ B activation in LPL/WM.

To our knowledge, this is the first report of the constitutional activation of $\mathrm{NF}-\kappa \mathrm{B}$ in LPL/WM. A previous study demonstrated cytoplasmic but no 
nuclear staining for p65 and p50 in 95\% of the 40 cases of LPL/WM studied. ${ }^{37}$ The reason for the discrepancy between their results and our own is unclear. A different polyclonal antibody used and/ or different antibody concentrations are possible explanations. On the assumption that unequivocal nuclear staining indicates NF- $\kappa \mathrm{B}$ activation, we found positive immunostaining in only a small subset of the neoplastic cells in many neoplasms.

In summary, we identified nuclear expression of BCL-10 in over half of cases of LPL/WM. BCL-10 nuclear expression was not associated with specific chromosomal abnormalities, and in particular, was not associated with chromosomal translocations that correlate with nuclear BCL-10 expression in MALT lymphomas. BCL-10 appears to have a biological role in a subset of LPL/WM because NF- $\kappa \mathrm{B}$ is activated in at least one third of cases. There was no correlation between BCL-10 and p65 NF- $\kappa$ B nuclear expression. However, the strong association of nuclear BCL-10 staining with extent of BM involvement, also possibly associated with nuclear p65 expression, may point toward a possible role of these two molecules in disease progression. It has recently been shown that MALT1 contains nuclear export signals and regulates the nucleocytoplasmic shuttling of the MALT1 and BCL-10 complex. $^{38}$ Nagakawa et $a l^{38}$ suggested that these molecules are involved not only in the NF- $\kappa \mathrm{B}$ pathway but in other biologic functions in lymphocytes. These results also raise the possibility that BCL-10 may be a potential therapeutic target in patients with LPL/WM.

\section{Acknowledgements}

We thank Dr Lynne Abruzzo for providing cytogenetic data. We also wish to thank Chris Yeager for his editorial suggestions and Ana M Martinez for her secretarial support.

\section{References}

1 Owen RG, Treon SP, Al-Katib A, et al. Clinicopathological definition of Waldenstrom's macroglobulinemia: consensus panel recommendations from the Second International Workshop on Waldenstrom's Macroglobulinemia. Semin Oncol 2003;30:110-115.

2 Jaffe ES, Harris NL, Stein H, et al. World Health Organization Classification of Tumors: Pathology and Genetics of Tumors of Haematopoietic and Lymphoid Tissues. IARC Press: Lyon, France, 2001.

3 Owen RG, Parapia LA, Higginson J, et al. Clinicopathological correlates of IgM paraproteinemias. Clin Lymphoma 2000;1:39-43; discussion 4-5.

4 Owen RG, Barrans SL, Richards SJ, et al. Waldenstrom macroglobulinemia. Development of diagnostic criteria and identification of prognostic factors. Am J Clin Pathol 2001;116:420-428.

5 Dimopoulos MA, Panayiotidis P, Moulopoulos LA, et al. Waldenstrom's macroglobulinemia: clinical fea- tures, complications, and management. J Clin Oncol 2000;18:214-226.

6 A clinical evaluation of the International Lymphoma Study Group classification of non-Hodgkin's lymphoma. The Non-Hodgkin's Lymphoma Classification Project. Blood 1997;89:3909-3918.

7 Dimopoulos MA, Alexanian R. Waldenstrom's macroglobulinemia. Blood 1994;83:1452-1459.

8 Owen RG, Johnson SA, Morgan GJ. Waldenstrom's macroglobulinaemia: laboratory diagnosis and treatment. Hematol Oncol 2000;18:41-49.

9 Bartl R, Frisch B, Mahl G, et al. Bone marrow histology in Waldenstrom's macroglobulinaemia. Clinical relevance of subtype recognition. Scand J Haematol 1983; 31:359-375.

10 Morel P, Monconduit M, Jacomy D, et al. Prognostic factors in Waldenström macroglobulinemia: a report on 232 patients with the description of a new scoring system and its validation on 253 other patients. Blood 2000;96:852-858.

11 Lin P, Medeiros LJ. Lymphoplasmacytic lymphoma/ waldenstrom macroglobulinemia: an evolving concept. Adv Anat Pathol 2005;12:246-255.

12 Kyle RA, Treon SP, Alexanian R, et al. Prognostic markers and criteria to initiate therapy in Waldenstrom's macroglobulinemia: consensus panel recommendations from the Second International Workshop on Waldenstrom's Macroglobulinemia. Semin Oncol 2003;30:116-120.

13 Lin P, Mansoor A, Bueso-Ramos C, et al. Diffuse large B-cell lymphoma occurring in patients with lymphoplasmacytic lymphoma/Waldenstrom macroglobulinemia. Clinicopathologic features of 12 cases. Am J Clin Pathol 2003;120:246-253.

14 Thome M. CARMA1, BCL-10 and MALT1 in lymphocyte development and activation. Nat Rev Immunol 2004;4:348-359.

15 Zhang Q, Siebert R, Yan M, et al. Inactivating mutations and overexpression of BCL10, a caspase recruitment domain-containing gene, in MALT lymphoma with $t(1 ; 14)(p 22 ; q 32)$. Nat Genet 1999;22: 63-68.

16 Willis TG, Jadayel DM, Du MQ, et al. Bcl10 is involved in $\mathrm{t}(1 ; 14)(\mathrm{p} 22 ; \mathrm{q} 32)$ of MALT B cell lymphoma and mutated in multiple tumor types. Cell 1999;96: $35-45$.

17 Ohshima K, Kawasaki C, Muta H, et al. CD10 and Bcl10 expression in diffuse large B-cell lymphoma: CD10 is a marker of improved prognosis. Histopathology 2001;39:156-162.

18 Ye H, Dogan A, Karran L, et al. BCL10 expression in normal and neoplastic lymphoid tissue. Nuclear localization in MALT lymphoma. Am J Pathol 2000; 157:1147-1154.

19 Ye H, Liu H, Attygalle A, et al. Variable frequencies of $t(11 ; 18)(q 21 ; q 21)$ in MALT lymphomas of different sites: significant association with CagA strains of $\mathrm{H}$ pylori in gastric MALT lymphoma. Blood 2003;102: 1012-1018.

20 Ye H, Gong L, Liu H, et al. MALT lymphoma with $\mathrm{t}(14 ; 18)(\mathrm{q} 32 ; \mathrm{q} 21) / \mathrm{IGH}-\mathrm{MALT} 1$ is characterized by strong cytoplasmic MALT1 and BCL10 expression. J Pathol 2005;205:293-301.

21 Kucharczak J, Simmons MJ, Fan Y, et al. To be, or not to be: NF-kappaB is the answer-role of Rel/NF-kappaB in the regulation of apoptosis. Oncogene 2003;22: 8961-8982. 
22 Takada Y, Singh S, Aggarwal BB. Identification of a p65 peptide that selectively inhibits NF-kappaB activation induced by various inflammatory stimuli and its role in down-regulation of NF-kappaBmediated gene expression and up-regulation of apoptosis. J Biol Chem 2004;279:15096-15104.

23 Bharti AC, Shishodia S, Reuben JM, et al. Nuclear factor-kappaB and STAT3 are constitutively active in CD138+ cells derived from multiple myeloma patients, and suppression of these transcription factors leads to apoptosis. Blood 2004;103:3175-3184.

24 Bueso-Ramos CE, Rocha FC, Shishodia S, et al. Expression of constitutively active nuclear-kappa B RelA transcription factor in blasts of acute myeloid leukemia. Hum Pathol 2004;35:246-253.

25 Biswas DK, Shi Q, Baily S, et al. NF-kappa B activation in human breast cancer specimens and its role in cell proliferation and apoptosis. Proc Natl Acad Sci USA 2004;101:10137-10142.

26 Sweeney C, Li L, Shanmugam R, et al. Nuclear factor$\mathrm{kappaB}$ is constitutively activated in prostate cancer in vitro and is overexpressed in prostatic intraepithelial neoplasia and adenocarcinoma of the prostate. Clin Cancer Res 2004;10:5501-5507.

27 Ye H, Chuang SS, Dogan A, et al. $\mathrm{t}(1 ; 14)$ and $\mathrm{t}(11 ; 18)$ in the differential diagnosis of Waldenstrom's macroglobulinemia. Mod Pathol 2004;17:1150-1154.

28 Achuthan R, Bell SM, Carr IM, et al. BCL10 in malignant lymphomas-an evaluation using fluorescence in situ hybridization. J Pathol 2002;196: 59-66.

29 Adachi A, Tamaru JI, Kaneko K, et al. No evidence of a correlation between BCL10 expression and API2-
MALT1 gene rearrangement in ocular adnexal MALT lymphoma. Pathol Int 2004;54:16-25.

30 Maes B, Demunter A, Peeters B, et al. BCL10 mutation does not represent an important pathogenic mechanism in gastric MALT-type lymphoma, and the presence of the API2-MLT fusion is associated with aberrant nuclear BCL10 expression. Blood 2002;99:1398-1404.

$31 \mathrm{Ye} \mathrm{H}$, Liu H, Raderer $\mathrm{M}$, et al. High incidence of $\mathrm{t}(11 ; 18)(\mathrm{q} 21 ; \mathrm{q} 21)$ in Helicobacter pylori-negative gastric MALT lymphoma. Blood 2003;101:2547-2550.

32 Sagaert X, Laurent $M$, Baens $M$, et al. MALT1 and BCL10 aberrations in MALT lymphomas and their effect on the expression of BCL10 in the tumour cells. Mod Pathol 2006;19:225-232.

33 Hayden MS, Ghosh S. Signaling to NF-kappaB. Genes Dev 2004;18:2195-2224.

34 Bonizzi G, Karin M. The two NF-kappaB activation pathways and their role in innate and adaptive immunity. Trends Immunol 2004;25:280-288.

35 Greten FR, Karin M. The IKK/NF-kappaB activation pathway-a target for prevention and treatment of cancer. Cancer Lett 2004;206:193-199.

36 Senftleben U, Cao Y, Xiao G, et al. Activation by IKKalpha of a second, evolutionary conserved, NFkappa B signaling pathway. Science 2001;293:14951499.

37 Leitch D, Barrans SL, Jack AS, et al. Dysregulation of apoptosis in Waldenstrom's macroglobulinemia does not involve nuclear factor kappa B activation. Semin Oncol 2003;30:161-164.

38 Nakagawa M, Hosokawa Y, Yonezumi M, et al. MALT1 contains nuclear export signals and regulates cytoplasmic localization of BCL10. Blood 2005;106:4210-4216. 